DOI 10. 18307/2018. 0604

(C) 2018 by Journal of Lake Sciences

\title{
湖泊污染底泥疏浚后新生沉积物一水界面溶解氧的动态响应”
}

\author{
古小治 ${ }^{1}$, 姜维华 ${ }^{2}$ \\ (1: 中国科学院南京地理与湖泊研究所湖泊与环境国家重点实验室,南京 210008) \\ (2:武进林业工作站, 常州 213161)
}

摘 要: 借助微氧电极测试技术对太湖贡湖湾试验区疏浚后的新生界面溶解氧动态进行一年的跟踪调研, 分析溶解氧在 新生微米级界面的分布特征、扩散通量以及界面附近有机质矿化速率. 结果表明疏浚后半年内, 溶解氧在表层沉积物的 侵蚀深度增大, 氧化层明显加厚. 氧气在新生界面表层沉积物中呈指数下降, 但衰减相对较缓. 在连续一年的跟踪调查中 发现, 仅秋季新生界面附近溶解氧浓度明显高于对照, 而在其他月份无差异. 污染底泥疏浚后一个月内氧扩散通量及有 机碳矿化速率下降最为明显, 仅为疏浚前的 $13 \%$, 其他月份沉积物一水界面氧的扩散通量、氧气的消耗速率、有机碳的降 解速率均有不同程度下降, 疏浚后新生界面氧气交换速率下降以及由此导致的有机碳矿化过程变缓可能深刻影响界面 生源要素的迁移过程.

关键词: 沉积物; 新生界面;氧气; 微电极; 疏浚; 太湖; 贡湖湾

\section{Dissolved oxygen dynamic across the new-born interface of bottom water and sediment after dredging contaminated sediment}

\author{
GU Xiaozhi ${ }^{1} \&$ JIANG Weihua ${ }^{2}$ \\ (1: State Key Laboratory of Lake Science and Environment, Nanjing Institute of Geography and Limnology, Chinese Academy \\ of Sciences, Nanjing 210008, P.R.China) \\ (2: Wujin Forest Protection Station, Changzhou 213161, P.R. China)
}

\begin{abstract}
Oxygen dynamic had been conducted a yearlong investigation to obtain oxygen distribution, oxygen diffusion flux and mineralization rate of organic matter across the new-born interface of bottom water and sediments due to dredge. The results showed that oxygen penetration depth increased with thickening oxidation layer in new-born sediments. Oxygen concentrations decreased rapidly with depth in the sediments, but relatively moderate. Below the penetration depth, the $\mathrm{O}_{2}$ concentration inside the sediment was under the detection limit (approximation for 0). Oxygen concentration in bottom water in autumn was significantly higher in dredge treatment than those in control, however, there was not statistically significant among the three sampling during the investigation. The diffusive $\mathrm{O}_{2}$ flux $\left(F_{\mathrm{O}_{2}}\right)$ and the oxygen consumption rate $\left(R_{\mathrm{O}_{2}}\right)$ were observed based on $\mathrm{O}_{2}$ microprofiles, otherwise, organic carbon degradation rates $\left(R_{\text {org }}\right)$ were obtained from the oxygen consumption rate based on the modified Redfield ratio $\left(\mathrm{O}_{2} /\right.$ $\mathrm{C}_{\text {org }}=170 / 117$ ) and the values also obviously decreased. Therefore, thickening oxidation layer and slowdown organic carbon degradation rates could significantly influence nutrients biogeochemical cycle after dredge.

Keywords: Sediment; new-born interface; oxygen; microelectrode; dredge; Lake Taihu; Gonghu Bay
\end{abstract}

目前, 针对湖泊内源污染的治理主要采用的方法是底泥疏浚、底泥覆盖、引水清污等物理方法以及种植 水生植物等生物方法 ${ }^{[1]}$. 底泥疏浚因能从湖体将污染物从底泥中永久性去除, 因而被认为是湖泊治理中的 重要工程措施而被广泛应用 ${ }^{[2-3]}$. 其中一些湖泊疏浚后在短期内对营养盐有较好的控制效果 (如滇池的草 海、杭州西湖、荷兰的 Kelemeer 湖等), 但随时间推移, 一些湖泊疏浚后良好的水质并不能长期保持, 主要反 映在湖泊的目标污染物浓度上升, 甚至出现疏浚后个别水体与原来相比反而增加 ${ }^{[3-5]}$. 其中疏浚后改变了原

* 国家自然科学基金面上项目 (41673123) 和天津市科技计划项目 (16YFXTSF00550) 联合资助. 2018-02-07 收稿; 2018-03-20 收修改稿. 古小治(1979 ), 男,博士, 副研究员;E-mail:xzgu@ niglas.ac.cn. 
来沉积物的物理、化学及生物学条件, 尤其是沉积物一水界面溶解氧的分布状况, 界面附近的溶解氧浓度, 对 沉积物中营养物释放具有控制作用,这些环境因子的改变可能会改变沉积物一水界面原有的营养盐循环模 式. 疏浚后新生表层的水一土界面会发生扩散、沉淀和溶解以及吸附和解析等许多瞬时过程, 这些过程对营 养盐在固相和水相的分配起着重要的作用, 如疏浚后新生表层的铁氧化物对磷有短时效的吸附作用, 疏浚 后间隙水中磷浓度立即减少, 底层上覆水中磷浓度增加, 疏浚后物质在河床的重建可能在较短时段 ( 分钟到 小时的尺度) 内完成 ${ }^{[6]}$.

底泥在湖泊内源负荷形成机制中具有重要的作用,其形成过程受到底泥与间隙水和上覆水之间复杂的 物理、化学和生物过程的影响. 氧气作为主要的生源要素, 其在上覆水体和沉积物中氧浓度变化动力学主要 由扩散、对流转换过程控制, 在稳态条件下, 溶解氧传输主要由扩散过程来控制, 而孔隙水和上覆水体间形 成的浓度梯度是氧气迁移的主要驱动力, 其在沉积物一水界面的分布及交换速率直接影响界面物质的赋存 及交换通量. 本文通过对太湖贡湖湾底泥疏浚后新生界面溶解氧动态的长期研究, 跟踪调查溶解氧的跨界 面分布与迁移过程, 以及定量评估表层有机质矿化速率影响, 以期为湖泊污染底泥工程治理进行科技支撑 和技术指导.

\section{1 材料与方法}

\section{1 研究区域概况}

研究区域位于太湖贡湖湾北部无锡市华庄附近, 该研究区域水体溶解氧、高锰酸盐指数、氨氮、总氮浓 度一直处于在 $\mathrm{I}$ 类 劣 $\mathrm{V}$ 类, 综合指标评价属地表水劣 $\mathrm{V}$ 类, 为全贡湖水质最差的水域 ${ }^{[7]}$. 针对水质目前状 况, 针对污染水域, 采用污染底泥环保疏浚方法控制内源污染, 底泥环保疏浚试验工程区总面积达 $0.10 \mathrm{~km}^{2}$, 环保疏浚时间为 2008 年 4-5 月. 在疏浚后一年内对疏浚区内新生表层沉积物一水界面氧气动态进行连续 跟踪监测.

\section{2 沉积物的采集及预处理}

研究针对工程实施前后的对照区和疏浚区进行一年的跟踪调研. 实验从 2008 年 3 月至 2009 年 6 月共 采集 4 次样品, 每研究区随机取 3 个平行柱状样, 柱状沉积物用装有有机玻璃管 $(\varphi 85 \mathrm{~mm} \times 600 \mathrm{~mm})$ 的柱状 采泥器采集, 泥层厚度不少于 $25 \mathrm{~cm}$, 上下加胶塞密封 (完整未扰动的柱样及其上覆水) 后, 垂直放置, 小心运 回实验室进行静态培养实验. 实验结束后按照设计要求进行柱样分层, 分层采用上顶法, 即用顶杆将底泥从 柱样底部向上小心顶出, 溢除上覆水后, 用切板切除. 将长度为 $20 \mathrm{~cm}$ 的柱样 按 $0 \sim 2 、 2 \sim 5 、 5 \sim 10$ 和 $10 \sim 20 \mathrm{~cm}$ 分样, 共 4 层, 湿底泥样经摚拌均匀后, 分析 烧失重、孔隙率、水分含量等. 另取出部分底泥在室温阴凉处干燥处理, 剩余 部分在冰柜中密封冷冻保存. 经干燥处理的底泥去除动植物碎屑后用研钵研 磨至 100 目, 待进一步分析总磷 $(\mathrm{TP})$ 、总氮 $(\mathrm{TN})$ 等参数, 其浓度测定采用碱 性过硫酸钾氧化-紫外/可见分光光度法.

\section{3 沉积物一水界面溶解氧浓度测定及耗氧速率计算}

1.3.1 沉积物一水界面溶解氧浓度的测定 水土界面氧气的垂向分布采用溶 氧微电极 (图 1) 进行 (PreSens, 德国) 测定. 该微电极的有效测定区 (sensing tip) 小于 $20 \mu \mathrm{m}$, 涂有固态光敏苂光粉; 在 $0 \% \sim 100 \%$ 空气饱和度水体中, 氧 气浓度与光强呈线性关系; 电极反应时间小于 $1 \mathrm{~s}$; 每次使用前, 采用饱和 空气 ( $100 \%$ 氧气) 与加人 $\mathrm{Na}_{2} \mathrm{SO}_{3}$ 的纯净水 $(0 \%$ 氧气) 进行两点校准. 电极安 装在微电极操作器 (专利 ZL200720043928.3) 上, 每秒下移电极 $0.037 \mathrm{~mm}$ 进 行连续测定, 直至氧气浓度接近于 $0.00 \mathrm{mg} / \mathrm{L}$ 为止.

1.3.2 氧气的扩散通量模型 在稳态情况下, 沉积物一上覆水界面的分布主要 受一维垂向传输和反应过程控制, 界面的交换过程以扩散为主. 溶解氧在沉 积物一上覆水界面的交换通量, 可用下式来计算 ${ }^{[8]}$ :

$$
F_{\mathrm{O}_{2}}=2 \varphi_{0} \cdot D_{\mathrm{s}} \cdot\left[O_{2}\right]_{\mathrm{BW}} / L
$$

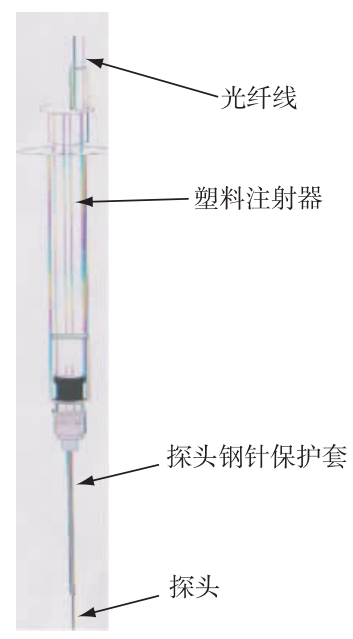

图 1 探针式微氧电极示意

Fig.1 Sketch of needle-type $\mathrm{O}_{2}$ microoptode 
式中, $F$ 为沉积物一上覆水界面扩散通量 $\left(\mathrm{mmol} /\left(\mathrm{m}^{2} \cdot \mathrm{d}\right)\right) ; \varphi_{0}$ 为表层沉积物 $(0 \sim 5 \mathrm{~mm})$ 的孔隙度, $D_{\mathrm{s}}$ 为考虑 了沉积物弯曲效应的实际分子扩散系数; $\left[O_{2}\right]_{\mathrm{BW}}$ 为底层水体氧气浓度 $(\mu \mathrm{mol} / \mathrm{L}) ; L$ 为氧气在沉积物中渗透 深度 $(\mathrm{mm})$. Ullman 等曾经给出了真实扩散系数 $D_{\mathrm{s}}$ 与孔隙度 $\varphi$ 之间的经验关系式 ${ }^{[9]}$ :

$$
D_{\mathrm{s}}=\varphi \cdot D_{0}(\varphi<0.7), D_{\mathrm{s}}=\varphi^{2} \cdot D_{0}(\varphi>0.7)
$$

式中, $D_{0}$ 为无限稀释溶液的理想扩散系数, 在 $25^{\circ} \mathrm{C}$, 对于 $\mathrm{O}_{2}, D_{0}=1.13 \times 10^{-9} \mathrm{~m}^{2} / \mathrm{s}^{[10]}$. 通过测定表层沉积物 $(0 \sim 5 \mathrm{~mm})$ 的平均孔隙度 $\varphi$ 得到 $D_{\mathrm{s}}$ 的数值.

1.3.3 氧气的扩散速率及有机质的降解速率 在稳态情况下, 剖面中氧气的扩散迁移速率和氧气的消耗速率 $\left(R_{\mathrm{O}_{2}}\right)$ 之间保持动态平衡, 也即:

$$
\frac{\partial F}{\partial Z}-R_{\mathrm{O}_{2}}=0
$$

由于沉积物中氧气的消耗与沉积物中易降解有机质密切相关, 如果忽略黄铁矿存在的情况下, 可利用 氧气的扩散通量计算出氧气的消耗速率 (或呼吸速率、呼吸商) 及初步估算出有机质的降解速率 $\left(C_{0_{2}} / C_{\text {org }}=\right.$ $138 / 106)^{[11-12]}$. 具体如下:

即

$$
\begin{aligned}
F_{\mathrm{O}_{2}} & =\int_{z=0}^{\infty} R_{\mathrm{O}_{2}} \mathrm{~d} z \\
F_{\mathrm{O}_{2}} & =R_{\mathrm{O}_{2}} \cdot z_{\mathrm{s}} \\
R_{\mathrm{O}_{2}} & =1.3 R_{\mathrm{org}}
\end{aligned}
$$

式中, $R_{\mathrm{O}_{2}}$ 为氧气的消耗速率 $\left(\mu \mathrm{mol} /\left(\mathrm{m}^{3} \cdot \mathrm{d}\right)\right) ; R_{\mathrm{org}}$ 为有机质的降解速率 $\left(\mu \mathrm{mol} /\left(\mathrm{m}^{3} \cdot \mathrm{d}\right)\right) ; z_{\mathrm{s}}$ 为氧气在沉积物 中的渗透深度 $(\mathrm{mm})$.

\section{2 结果与讨论}

\section{1 疏浚对沉积物物化特性的影响}

疏浚前后, 沉积物的水分含量、烧失重及孔隙度自上而下均呈明显的减少趋势. 在疏浚后表层 $0 \sim 2 \mathrm{~cm}$ 水分含量最高, 主要为水体再悬浮颗粒物沉积物层, 较亚表层沉积物水分含量高 $5 \%$, 越往下层沉积物水分 含量越接近疏浚前对照区心土层数值, 变化越不明显 (表 1). 孔隙度变化趋势与水分含量一致. 当表层水分 含量相对较高的 $20 \mathrm{~cm}$ 沉积物被疏浚后, 相对紧实度较高的亚表层沉积物成为新生表层, 它的水分含量及孔 隙度则较低, 孔隙度和含水率是底泥固体颗粒之间空间大小的衡量指标. 底泥孔隙之间填充着间隙水溶液 或者气体物质, 孔隙度的大小直接决定了底泥间隙水中营养盐的扩散能力, 与底泥中物质的释放有密切关 系 ${ }^{[13]}$. 孔隙度越大则营养盐扩散的弯曲度越小, 扩散能力就越强 ${ }^{[14]}$.

表 1 太湖贡湖湾采样点沉积物的基本性质

Tab.1 Primary property in the sediments of study areas in Gonghu Bay of Lake Taihu

\begin{tabular}{ccccccccc}
\hline & 剖面 & $\begin{array}{c}\text { 水分含量/ } \\
\%\end{array}$ & $\begin{array}{c}\text { 烧失重/ } \\
\left(\mathrm{cm}^{3} / \mathrm{g}\right)\end{array}$ & $\begin{array}{c}\text { 孔隙度/ } \\
\%\end{array}$ & $\begin{array}{c}\mathrm{TOC} / \\
\%\end{array}$ & $\begin{array}{c}\mathrm{TN} / \\
(\mathrm{mg} / \mathrm{kg})\end{array}$ & $\begin{array}{c}\mathrm{TP} / \\
(\mathrm{mg} / \mathrm{kg})\end{array}$ & $\mathrm{C} / \mathrm{N}$ \\
\hline \multirow{2}{*}{ 疏浚区 } & $0 \sim 2 \mathrm{~cm}$ & 55.05 & 5.37 & 75.38 & $0.76 \pm 0.06$ & $1485 \pm 98$ & $486 \pm 45$ & $5.04 \sim 5.17$ \\
& $2 \sim 5 \mathrm{~cm}$ & 52.66 & 5.13 & 73.55 & $1.19 \pm 0.03$ & $1620 \pm 109$ & $520 \pm 56$ & $7.07 \sim 7.70$ \\
& $5 \sim 10 \mathrm{~cm}$ & 48.62 & 4.48 & 70.29 & $1.34 \pm 0.07$ & $1301 \pm 78$ & $703 \pm 54$ & $10.25 \sim 10.42$ \\
& $10 \sim 20 \mathrm{~cm}$ & 46.86 & 3.64 & 68.80 & $2.07 \pm 0.05$ & $1382 \pm 90$ & $432 \pm 34$ & $14.38 \sim 15.61$ \\
对照区 & $0 \sim 2 \mathrm{~cm}$ & 72.60 & 7.66 & 86.88 & $1.41 \pm 0.10$ & $2482 \pm 67$ & $641 \pm 52$ & $4.18 \sim 4.74$ \\
& $2 \sim 5 \mathrm{~cm}$ & 68.76 & 4.90 & 84.62 & $1.26 \pm 0.08$ & $2167 \pm 89$ & $571 \pm 42$ & $5.67 \sim 5.93$ \\
& $5 \sim 10 \mathrm{~cm}$ & 67.48 & 4.26 & 83.84 & $1.55 \pm 0.06$ & $1970 \pm 96$ & $499 \pm 26$ & $7.81 \sim 7.97$ \\
& $10 \sim 20 \mathrm{~cm}$ & 58.47 & 3.58 & 77.88 & $1.31 \pm 0.08$ & $1798 \pm 79$ & $537 \pm 31$ & $7.16 \sim 7.40$ \\
\hline
\end{tabular}

由于 $\mathrm{NH}_{4}^{+}$和 $\mathrm{PO}_{4}^{3-}$ 在沉积物水界面扩散以分子扩散为主, 而孔隙度与扩散通量之间呈正比 ${ }^{[14]}$, 沉积物孔 隙度降低势必降低沉积物间隙水中 $\mathrm{NH}_{4}^{+}$和 $\mathrm{PO}_{4}^{3-}$ 的释放通量, 这在一定程度上可抑制沉积物间隙水中营养盐 
的释放,对控制湖泊内源营养盐污染至关重要. TOC 含量在剖面分布上由表层向底层逐步降低, 而 TN 和 TP 含量分布趋势基本一致, 表层高于底层. 研究区域沉积物 $\mathrm{C} / \mathrm{N}$ 主要分布在 5.04 15.61 之间. $\mathrm{C} / \mathrm{N}$ 通常被作 为识别有机质来源和类型的一个重要指标, $\mathrm{C} / \mathrm{N}$ 越高有机质就越难降解, 比值在 5 6 时, 被认为是新鲜的 或易降解的有机质组分; 当比值高于 10 时, 被认为是难降解有机质组分 ${ }^{[16-17]}$. 发现研究的疏浚区域表层 $0 \sim$ $2 \mathrm{~cm}$ 的沉积物 $\mathrm{C} / \mathrm{N}$ 大部分介于 $5 \sim 6$ 之间, 据现场采样观测, 它们主要由死亡的水草、腐烂的蓝藻及悬浮的 表层有机颗粒物, 这部分物质均由易降解有机物质组成, 由于它们直接与上覆水体接触, 对上覆水体氮磷影 响可能较大, 尤其是对照区,TOC、TN 及 TP 含量远高于其他疏浚试验区.

\section{2 疏浚前后新生界面溶解氧的变化}

为了研究疏浚对微界面氧化还原状况的影响, 分别在疏浚前( 2008 年 3 月 16 日) 及疏浚后 (2008 年 6 月 11 日) 利用微氧电极分析了剖面溶解氧的分布状况 (图 2). 溶解氧浓度在上覆水 $1 \mathrm{~mm}$ 以上基本保持不 变, 在此深度以下溶解氧浓度急剧下降, 尤其是在沉积物中的含量呈指数下降, 具体遵循 $C_{z}=C_{0} \cdot \mathrm{e}^{-k z}$ 的分布, 式中, $C_{z}$ 为沉积物剖面中深度 $z$ 处溶解氧浓度 $(\mathrm{mg} / \mathrm{L}) ; C_{0}$ 为界面处溶解氧浓度 $(\mathrm{mg} / \mathrm{L}) ; k$ 为常数, 各回归方 程的决定系数 $R^{2}>0.85$, 均达到极显著水平. 分析溶解氧在沉积物中的含量发现, 疏浚前的试验区内半浓度 衰减深度 $Z_{0.5}$ 均没有显著差异 $(P>0.05)$. 然而疏浚前后 $Z_{0.5}$ 数值差异显著 $(P<0.05)$, 也即疏浚能显著提高表 层微界面附近沉积物中溶解氧浓度. 溶解氧在沉积物中渗透深度疏浚后的疏浚区、溶解氧的渗透深度 $z_{\mathrm{s}}$ 数 值较疏浚前该区显著提高 $(P<0.05)$. 另外疏浚措施也能显著提高界面处溶解氧浓度 $($ 表 2$)$, 这主要与表层 沉积物中易降解有机质被移走有关 ${ }^{[18-19]}$. 疏浚后, 通过对比试验区沉积物剖面 $\mathrm{O}_{2}$ 的分布状况, 也可看出疏浚 表层污染底泥后对沉积物剖面氧化还原状况改善是明显的,与对照区相比均有不同程度的提高.

然而溶解氧的大幅提高对沉积物界面氮磷营养盐的扩散迁移过程至关重要 ${ }^{[8,18-20]}$. 沉积物一水界面的 物理化学条件在控制磷的流量作用中比总溶解磷的浓度梯度更重要, 沉积物表层几毫米的氧化还原反应对 磷的化学行为起着重要的作用, 在好氧体系中, 沉积物氧化层的吸附和沉淀作用控制磷的交换, 而不是还原 层的扩散作用. 范成新等 ${ }^{[5]}$ 研究发现疏浚之所以能够有效控制内源污染, 在于疏浚后沉积物新生界面上形 成一较致密的氧化层, 该新生表层直接与氧含量较为充足的上覆水体接触, 使新生表层的氧化还原电位上 升, $\mathrm{Fe}^{2+}$ 被转化为 $\mathrm{Fe}^{3+}$, 再与孔隙水中游离的 $\mathrm{PO}_{4}^{3-}$ 结合, 形成 $\mathrm{FePO}_{4}$ 沉淀或铁氧化物吸附间隙水中 $\mathrm{PO}_{4}^{3-}$ 而 滞留在沉积物, 该氧化层起到了阻止下层孔隙水中磷的释放作用. 然而当氧化层很薄或不存在时, 从有机质 或铁氧化物还原释放出的磷就可释放到上覆水体中.

表 2 太湖贡湖湾试验区疏浚前后溶解氧在界面的扩散通量

Tab.2 Diffusive oxygen fluxes across sediments-water surface before and after areas dredged study of Gonghu Bay of Lake Taihu

\begin{tabular}{|c|c|c|c|c|c|c|c|c|}
\hline 处理 & 采样时间 & 拟合方程 & $R^{2}$ & $Z_{0.5} / \mathrm{mm}$ & $\begin{array}{l}{\left[\mathrm{O}_{2}\right] /} \\
(\mathrm{mg} / \mathrm{L})\end{array}$ & $\varphi$ & $\begin{array}{l}z_{\mathrm{s}} / \\
\mathrm{mm}\end{array}$ & $\begin{array}{c}F / \\
\left(\mathrm{mmol} /\left(\mathrm{m}^{2} \cdot \mathrm{d}\right)\right)\end{array}$ \\
\hline \multirow[t]{4}{*}{ 对照组 } & $2008-03-16$ & $C_{z}=14.77 \mathrm{e}^{-3.3 z}$ & 0.913 & 0.21 & 5.99 & 0.75 & 1.63 & 9.46 \\
\hline & $2008-06-11$ & $C_{z}=11.39 \mathrm{e}^{-3.56 z}$ & 0.932 & 0.19 & 5.28 & 0.81 & 1.59 & 10.77 \\
\hline & $2008-09-14$ & $C_{z}=6.535 \mathrm{e}^{-3.49 z}$ & 0.924 & 0.20 & 3.76 & 0.84 & 1.78 & $7.64^{*}$ \\
\hline & $2009-06-12$ & $C_{z}=10.82 \mathrm{e}^{-2.82 z}$ & 0.883 & 0.28 & 5.12 & 0.87 & 1.89 & 10.89 \\
\hline \multirow[t]{4}{*}{ 疏浚区 } & $2008-03-16$ & $C_{z}=23.72 \mathrm{e}^{-1.90 z}$ & 0.848 & $0.36^{*}$ & $7.57^{*}$ & 0.75 & $3.37 *$ & 5.78 \\
\hline & $2008-06-11$ & $C_{z}=5.415 \mathrm{e}^{-1.48 z}$ & 0.868 & $0.47^{*}$ & 3.55 & 0.81 & $3.62 *$ & $3.18^{*}$ \\
\hline & $2008-09-14$ & $C_{z}=17.89 \mathrm{e}^{-1.98 z}$ & 0.891 & $0.35^{*}$ & $5.46^{*}$ & 0.84 & $3.22 *$ & 6.13 \\
\hline & $2009-06-12$ & $C_{z}=9.518 \mathrm{e}^{-2.93 z}$ & 0.938 & 0.24 & 4.30 & 0.87 & 2.07 & 8.35 \\
\hline
\end{tabular}

$Z_{0.5}$ 为沉积物中氧气浓度衰减到界面处浓度 $\left[\mathrm{O}_{2}\right]$ 数值一半时沉积物中的渗透深度; $\varphi$ 为表层 $0 \sim 5 \mathrm{~mm}$ 沉积物孔隙度的平 均值; $z_{\mathrm{s}}$ 为氧气的渗透深度, 也即氧气在沉积物中浓度接近零时的深度; $*$ 表示经统计检验在 $P<0.05$ 水平上数值间有 显著差异.

疏浚后试验区的疏浚区溶解氧的扩散通量均有所下降, 其中刚疏浚后一个月内下降最为明显, 仅为疏 浚前的 $29.5 \%$, 与疏浚前各试验区氧气扩散通量相比显著下降 $(P<0.05)$ (表 2$)$. 对比氧气的消耗速率 $R_{\mathrm{O}_{2}}$ 及 

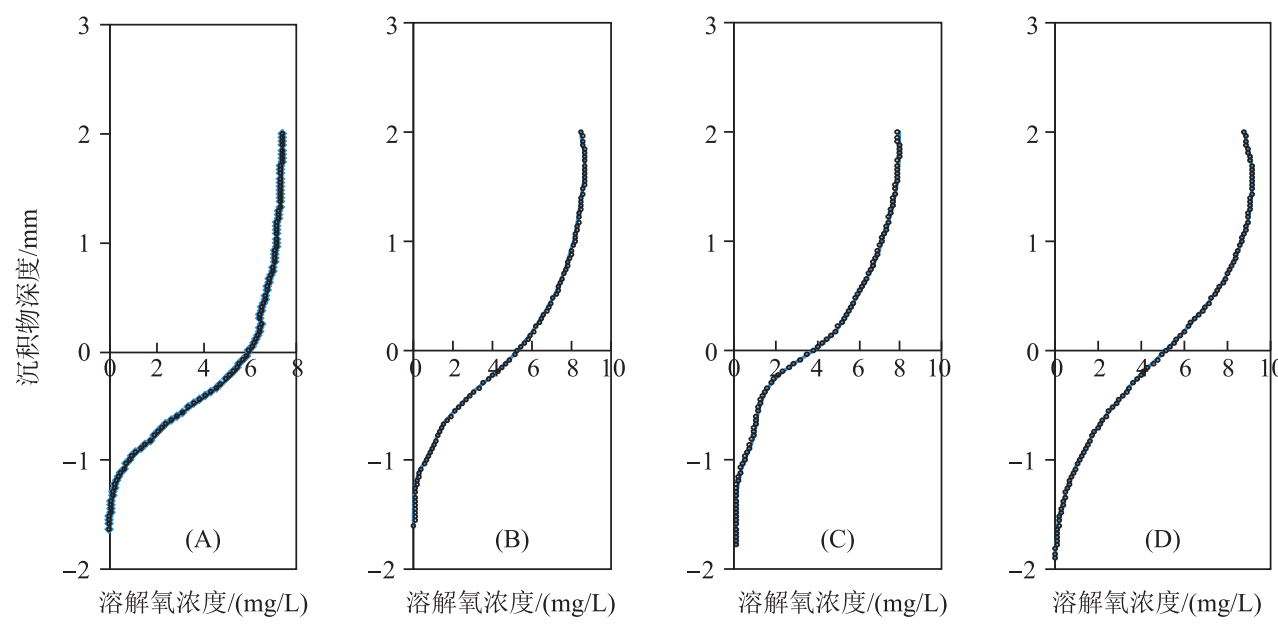

( I )
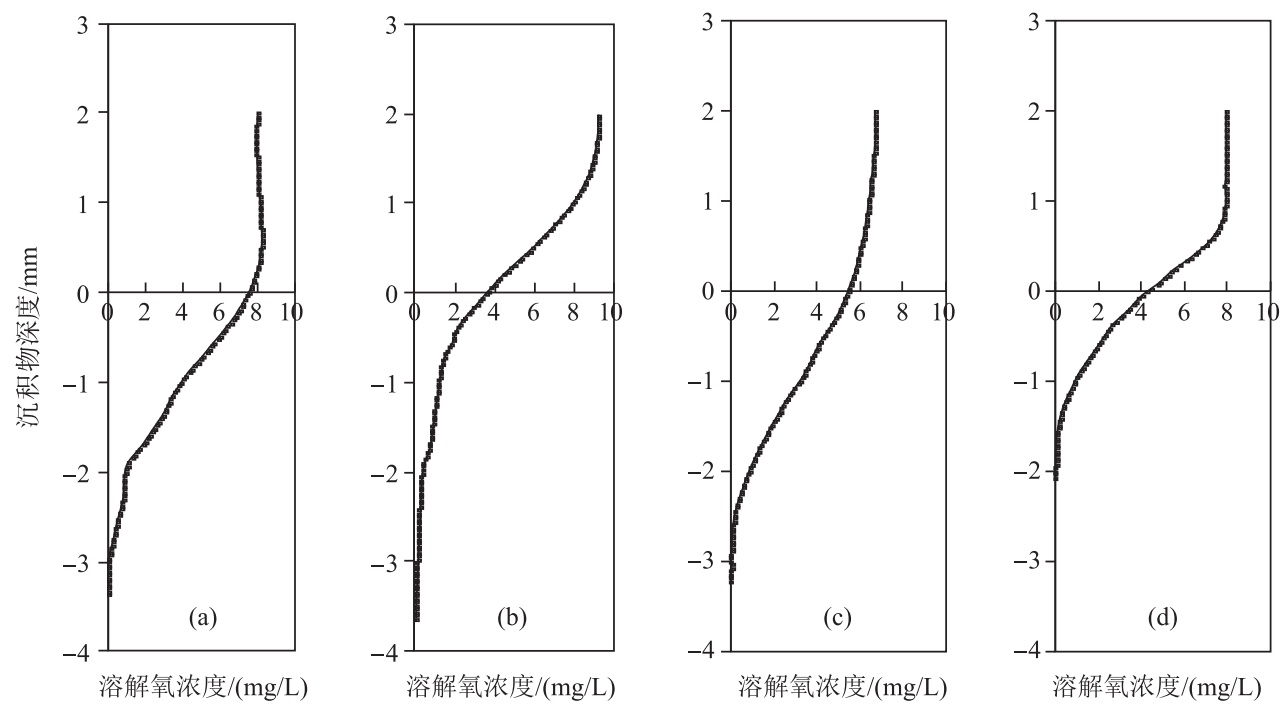

(II)

图 2 未疏浚的实验对照区 ( I ) 和疏浚区 (II) 在一周年沉积物一水微界面溶解氧浓度的垂向分布动态

( A , B , C , D 及 a, b , c, d 分别为 2008-03-16,2008-06-11,2008-09-14 和 2009-06-12 在对照区和 疏浚区采集柱状沉积物样品, 然后利用溶解氧微电极测定溶解氧浓度剖面分布)

Fig.2 Vertical concentration profiles of dissolved oxygen at sediments water surface before dredged( I ) and within dredged( II )

有机碳的降解速率 $R_{\text {org }}$ 均呈现出相同的趋势. 这说明疏浚对降低表层沉积物有机质的降解可起到很好的控 制作用, 而有机质也一般被认为是间隙水中氮、磷营养盐的主要来源 ${ }^{[21-22]}$.

由于沉积物中有机质的组成及各组分在不同代谢途径中的作用不同, 呼吸商数值在 $0.7 \sim 2.5$ 之间波 动 ${ }^{[23]}$, 本研究采用平均值 0.77 来计算有机质分解速率. 氧气消耗速率 $R_{0_{2}}$ 疏浚前为 $1.57 \sim 2.47 \mathrm{mmol} /\left(\mathrm{m}^{3} \cdot \mathrm{a}\right)$, 疏浚后为 $0.32 \sim 1.47 \mathrm{mmol} /\left(\mathrm{m}^{3} \cdot \mathrm{a}\right)$, 为疏浚前的 $13 \% \sim 70 \%$, 两者相比下降明显. 再进一步比较有机质的降 解速率 $R_{\text {org }}$, 疏浚前为 $14.48 \sim 22.84 \mathrm{mg} /\left(\mathrm{m}^{3} \cdot \mathrm{a}\right)$, 疏浚后为 $2.96 \sim 13.60 \mathrm{mg} /\left(\mathrm{m}^{3} \cdot \mathrm{a}\right)$, 为疏浚前的 $13 \% \sim 70 \%$ (图 3). 这与 Dedieu 等 ${ }^{[24]}$ 研究法国地中海沿岸沉积物及 Jørgensen 等 ${ }^{[25]}$ 和 Cai 等 ${ }^{[8]}$ 研究大陆架沉积物氧气 的消耗速率的计算结果接近.

在春、夏季之际, 浮游生物的迅速生长也可造成表层沉积物中易降解有机质的大量沉积, 但疏浚后沉积 


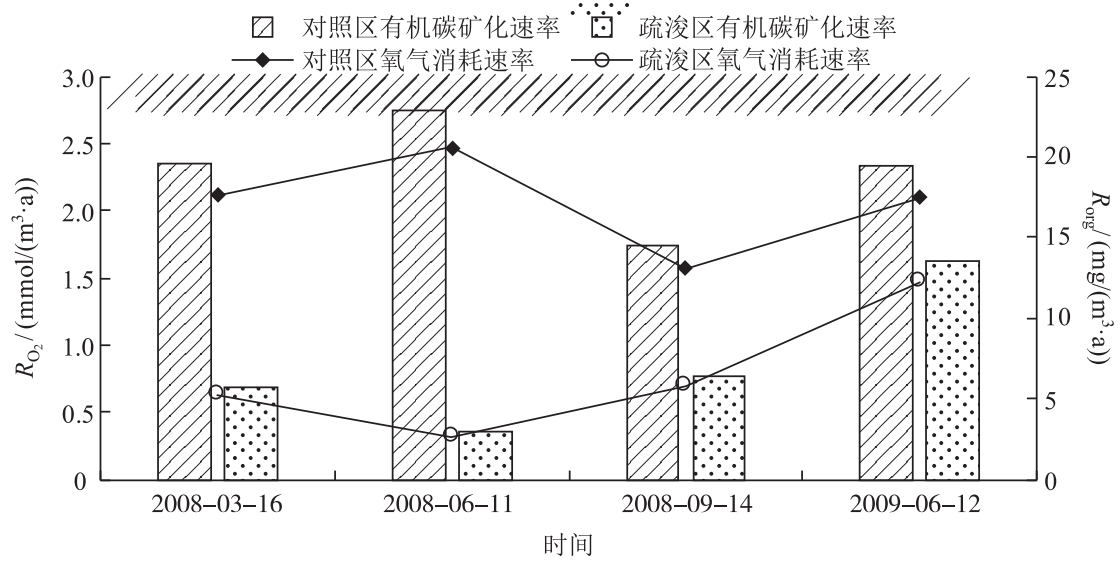

图 3 疏浚工程实施前后贡湖湾一年内沉积物一水界面处氧气的消耗速率及有机质降解速率动态

Fig.3 Oxygen consumption rate in surface sediments $\left(R_{\mathrm{O}_{2}}\right)$, and organic carbon degradation rate $\left(R_{\text {org }}\right)$ in study areas in Gonghu Bay after dredge

物氧化层厚度较对照有一个显著提升, 说明底泥疏浚改良措施在改善沉积物氧化状况中的作用是显著的. 另外沉积物中的厌氧代谢过程如反硝化过程虽然并未直接利用沉积物中氧气, 但它代谢的底物硝酸根主要 来源于氨氮经消化细菌氧化产生的硝酸根,这一氧化过程将消耗沉积物质约 $33 \%$ 的氧气 ${ }^{[26]}$. 为此根据文献 报道, 经验公式初步推算出试验区沉积物中反硝化速率: 反硝化速率 $=0.105 \times R_{0}$, 疏浚前和疏浚后试验区各 采样点沉积物反硝化速率分别为 $0.164 \sim 0.260$ 和 $0.034 \sim 0.155 \mathrm{mmol} /\left(\mathrm{m}^{3} \cdot \mathrm{a}\right)$. Berelson 等 ${ }^{[26]}$ 研究 Port Phillip 湾, 发现沉积物中营养盐的再生量占每年溶解氮总负荷的 63\%、占溶解性磷总负荷的 $72 \%$. 然而整个海湾沉 积物的反硝化损失量相当于可再循环氮的 $63 \%$. 对北大西洋 9 个河口系统氮元素收支的研究结果表明,进 人该生态系统的氮 $30 \% \sim 65 \%$ 经反硝化而损失,或被沉积物隔绝而不能进人邻近海区 ${ }^{[27-28]}$. 由此可进一步发 现疏浚能降低反硝化速率,减少反硝化过程产生等温室气体 $\mathrm{N}_{2} \mathrm{O}$ 的排放数量也相当可观.

\section{3 结论}

通过对太湖贡湖湾试验区疏浚后的新生界面溶解氧动态近一年的跟踪调研主要得出以下结论:

1) 疏浚工程措施能明显降低表层沉积物氮磷污染负荷, 另外表层沉积物中有机质主要为 $\mathrm{C} / \mathrm{N}$ 数值较高 的难降解组分,这在一定程度上可减少底层水体氮磷营养盐负荷水平.

2) 疏浚能显著改善界面及界面下表层沉积物的氧气状况. 疏浚后半年内,溶解氧在表层沉积物的侵蚀 深度显著增大, 另外氧气在新生界面下表层沉积物中衰减相对较缓. 另外疏浚工程在秋季可明显提高沉积 物一水微界面附近溶解氧浓度, 而在其他月份底层水体溶解氧无差异.

3) 疏浚能降低氧气在沉积物一水界面的氧气交换速率和减缓有机质矿化. 刚疏浚后的一个月内下降最 为明显 $(P<0.05)$, 氧扩散通量仅为疏浚前的 $13 \%$.

\section{4 参考文献}

[ 1 ] Li H, Zhou Q, An M. Study on the effective control of internal phosphorus loading in eutrophic water. Fujian Environment, 2003, 20(2) : 33-35. [李辉, 周琪, 安沝. 富营养化水体中内源磷负荷的有效控制. 福建环境, 2003, 20(2) : 33-35.]

[ 2 ] Zhong JC, Liu GF, Fan CX et al. Environmental effect of sediment dredging in lake (I) : the role of sediment dredging in reducing internal nitrogen release. J Lake Sci, 2009, 21(3): 335-344. DOI:10.18307/2009.0304. [钟继承, 刘国锋, 范成新等. 湖泊底泥疏浚环境效应: II. 内源氮释放控制作用. 湖泊科学, 2009, 21(3) : 335-344.]

[ 3 ] Zhong JC, Liu GF, Fan CX et al. Environmental effect of sediment dredging in lake ( I) : The role of sediment dredging in reducing internal phosphorous release. J Lake Sci, 2009, 21(1): 84-93. DOI: 10.18307/2009.0111. [钟继承, 刘国锋, 
范成新等. 湖泊底泥疏浚环境效应：I . 内源磷释放控制作用. 湖泊科学, 2009, 21(1) : 84-93.]

[ 4 ] Ruley JE, Rusch KA. An assessment of long-term post-restoration water quality trends in a shallow, subtropical, urban hypereutrophic lake. Ecological Enggineering, 2002, 19: 265-280.

[ 5 ] Fan CX, Zhang L, Qin BQ et al. Migration mechanism of biogenic elements and their quantification on the sediment-water interface of Lake Taihu: I. Spatial variation of the ammonium release rates and its source and sink fluxes. J Lake Sci, 2004, 16(1) : 11-21. DOI: 10.18307/2004.0102. [范成新, 张路, 秦伯强等. 太湖沉积物-水界面生源要素迁移机制 及定量化一1. 铵态氮释放速率的空间差异及源-汇通量. 湖泊科学, 2004, 16(1) : 10-20.]

[ 6 ] Falcão M, Gaspar MB, Caetano M et al. Short-term environmental impact of calm dredging in coastal waters( south of Portugal) : chemical disturbance and subsequent recovery of seabed. Marine Environmental Research, 2003, 56: 649-664.

[ 7 ] Xu ZA. Gong Lake Xidong waterworks water quality study [Dissertation]. Nanjing: Hohai University, 2005. [徐兆安. 贡 湖锡东水厂水质可达性研究 [学位论文]. 南京: 河海大学, 2005.]

[ 8 ] Cai WJ, Reimers CE. Benthic oxygen flux, bottom water oxygen concentration and core top organic carbon content in the deep northeast Pacific Ocean. Deep Sea Research Part I: Oceanographic Research Papers, 1995, 42(10) : 1681-1699.

[ 9 ] Ullman WS, Aller RC. Diffusion coefficients in nearshore marine sediments. Limnology and Oceanography, 1982, 27 : 552-556.

[10] Schulz H, Zabel M. Marine geochemistry, 1st edition. Netherlands: Springer, 2000.

[11] Froelich PN, Klinkhammer GP, Bender ML et al. Early oxidation of organic matter in pelagic sediments of the eastern equatorial Atlantic: suboxic diagenesis. Geochimica et Cosmochimica Acta, 1979, 43: 1075-1090.

[12] Berner RA. Early Diagenesis: A theoretical approach. Princeton: Princeton University Press, 1980.

[13] David B, Hutton EWH, James PM et al. Exponential approximation to compacted sediment porosity profiles. Computers Geosciences, 2001, 27 : 691-700.

[14] Krom MD, Berner RA. The diffusion coefficients of sulfate, ammonium and phosphate rons in anoxic marine sediments. Limnology and Oceanography, 1980, 25(2) : 327-337.

[15] Murray LG, Mudge SM, Newton A et al. The effect of benthic sediments on dissolved nutrient concentrations and fluxes. Biogeochemistry, 2006, 81: 159-178.

[16] Rasheed M. Nutrient Fluxes from sediments of the northern Gulf of Aqaba under various anthropogenic activities. Lebanese Sci J, 2004, 5: 3-16.

[17] Rasheed M, Badran MI, Huettel M. Influenceof sediment permeability and mineral composition on organic matter degradation in three sediments from the Gulf of Aqaba, Red Sea. Estuarine, Coastal and Shelf Science, 2003, 57: 369-384.

[18] Sauter EJ, Schlüter M, Suess E. Organic carbon flux and remineralization in surface sediments from the northern North Atlantic derived from pore-water oxygen microprofiles. Deep-Sea Research I, 2001, 48: 529-553.

[19] Paropkari AL, Prakash BC, Mascarenhas A. A critical evaluation of depositional parameters controlling the variability of organic carbon in Arabian Sea sediments. Marine Geology, 1992, 107(3) : 213-226.

[20] Glud RN, Gundersen JK, Røy H et al. Seasonal dynamics of benthic $\mathrm{O}_{2}$ uptake in a semi-enclosed bay : importance of diffusion and faunal activity. Limnology and Oceanography, 2003, 48(3) : 1265-1276.

[21] Witek Z, Drgas A, Ameryk A et al. Production and mineralization of organic matter in the Pomeranian Bay (Baltic Sea). Bulletin of the Sea Fisheries Institute, Gdynia, 2001, 154(3) : 49-69.

[22] Martin WR, Sayles FL. Organic matter cycling in sediments of the continental margin in the northwest Atlantic ocean. DeepSea Research I, 2004, 51: 457-489.

[23] Hopkinson CS, Giblin AE, Tucker J. Benthic metabolism and nutrient regeneration on the continental shelf of Eastern Massachusetts, USA. Marine Ecology Progress Series, 2001, 224: 1-19.

[24] Dedieu K, Rabouille $\mathrm{C}$, Thouzeau G et al. Benthic $\mathrm{O}_{2}$ distribution and dynamics in a Mediterranean lagoon (Thau, France) : An in situ microelectrode study. Estuarine, Coastal and Shelf Science, 2007, 72: 393-405.

[25] Jørgensen BB, Glud RN, Holby O. Oxygen distribution and bioirrigation in Arctic fjord sediments ( Svalbard, Barents Sea). Marine Ecology Progress Series, 2005, 292: 49-58.

[26] Berelson WM, Heggie D et al. Benthic nutrient recycling in Port Phillip Bay, Australia. Esturine, Coastal and Shelf Seienee, 1998, 46: 917-934.

[27] Boynton WR, Kemp WM. Nutrient regeneration and oxygen consumption by Sediment along an estuarine salinity gradient. Marine Ecology Progress Series, 1985, 23: 45-55.

[28] Sloth NP, Blackburn H, Las Stenvang H et al. Nitrogen cycling in sediments with different organic loading. Marine Ecology Progress Series, 1995, 116: 163-170. 